\title{
Nano-Focused Vibrational Spectroscopy Reaching the Single Quantum Level: Imaging Structure, Function, and Dynamics on the Nanoscale
}

\author{
Markus B. Raschke ${ }^{1}$
}

${ }^{1}$ Department of Physics, Department of Chemistry, and JILA, University of Colorado, Boulder, CO 80309, USA

Structure, function, and dynamics of many interfacial soft-matter systems, including polymer heterostructures, organic photovoltaics, or biomembranes are typically defined on the mesoscopic few $\mathrm{nm}$ to sub-micron scale. Tip-enhanced and scattering scanning near-field optical microscopy ( $s$-SNOM) has already demonstrated its ability to spectroscopically access that relevant spatial regime. In combination with IR-vibrational spectroscopy $s$-SNOM provides molecular structural information. We demonstrated how the combination of $s$-SNOM with plasmonic and optical antenna concepts and ultrafast and shaped laser pulses allows for the precise control of an optical excitation on femtosecond time and nanometer length scales extending $s$-SNOM into the nonlinear and ultrafast regime. I will then discuss how, from the multi-spectral combination of different coherent and incoherent light sources we gain insight into the microscopic intra- and intermolecular interaction. I will discuss several specific implementations from our recent work on block-copolymers, proteins, and self-assembled monolayers.

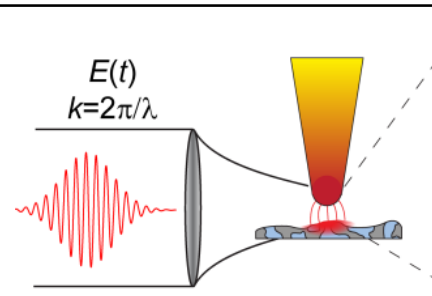

Far- to near-field transformation

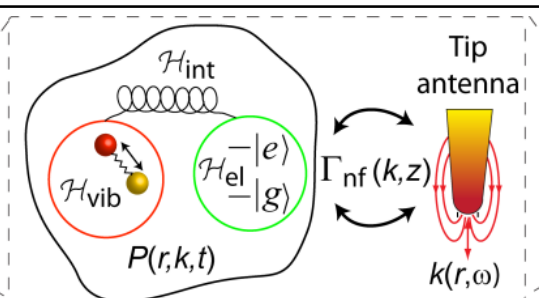

Near- to far-field transformation

Fig. 1 Far- to near-field transformation and interaction dynamics in ultrafast scattering-scanning near-field optical microscopy (s-SNOM). The light-matter interaction rate $\Gamma_{\mathrm{NF}}(\mathrm{k}, \mathrm{z})$ between antenna tip and the quantum emitter is enhanced via the large near-field k-vector distribution $\mathrm{k}(\mathrm{r}, \omega)$. The tip as optical antenna mediates the impedance matching between local and far-field electromagnetic density of states (LDOS) and gives rise to enhanced net excitation and emission rates.

The extension of ultrafast spectroscopy to the nanoscale to access coupling, dynamics, and interactions on the level of single quantum objects or quantum excitations has been a longstanding challenge. Access into this new ultrafast spectroscopic regime requires i) to overcome the spatial resolution limit of conventional far-field spectroscopy, and ii) to enhance the interaction rate for more efficient light-matter coupling. 
Combining plasmonic and optical antenna concepts with ultrafast and shaped laser pulses allows for the precise control of an optical excitation on femtosecond time and nanometer length scales. I will discuss concepts describing the induced near-field light-matter interaction in terms of impedance matching to a quantum system [1]. I will extend into questions concerning the competition of radiative and nonradiative decay of the metallic nanostructure against preserving and manipulating the quantum coherence of the coherent excitation. Specific examples from our lab include adiabatic nano-focusing on a tip for

nano-spectroscopy, spatio-temporal superfocusing, and optical control at the $10 \mathrm{~nm}-10$ fs level [2,3]. We demonstrate reaching the quantum regime at the tunneling limit. We furthermore demonstrate the optical antenna coupled ultrafast free-induction decay in near-field infrared nano-spectroscopy of vibrational resonances and both their tip-enhanced decoherence [4] as well as the observations of long-lived intrinsic coherences for small molecular ensembles. I will conclude with an outlook for quantum coherent control in the near-field, strong light-matter interaction, and new ways for radiative decay engineering complementing cavity optics.

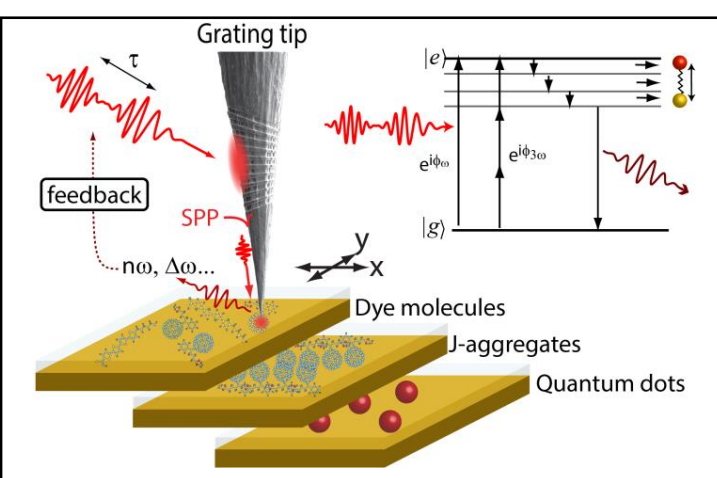

Fig 2. Example for eperimental implementation of nanofocused few-fs dynamics experiments. Pulse pairs are generated by a pulse shaper, with controllable delay and waveform, enabling coherent control through the phase relationship between excitation pathways (see band structure diagram, inset). Feedback for optimization is based on the luminescent or nonlinear response of the coupled tipsample system

\section{References}

[1] R. L. Olmon and M. B. Raschke, Nanotechnology 23, 444001 (2012).

[2] S. Berweger, J.M. Atkin, X.G. Xu, R.L. Olmon, and M.B. Raschke, Nano Lett. 11, 4309 (2011).

[3] V. Kravtsov, J.M. Atkin, and M.B. Raschke, Optics Lett. 38, 1322 (2013). [4] X.

G. Xu and M. B. Raschke, Nano Lett. 13, 1588 (2013). 